\title{
Research on the mathematical modeling Protodyakonov coefficient of coal and rock hardness based on shearer motors working parameters
}

\author{
Yang Jian-jian, Li Xu, Zhou Jianfeng, Jiang Hai, WU Miao \\ School of Mechanical, Electrical \& Information Engineering, China University of Mining and \\ Technology (Beijing), Beijing 100083, China \\ email: yangjiannedved@163.com
}

Keywords: Shearer; Coal rock hardness; Motor parameters; Mathematical model; Proportionality coefficient

\begin{abstract}
In view of the current issue that the shearer in coal mine fully mechanized working face can't accurate automatic identification of coal rock interface, the method of using the shearer motor working parameters to reflect the hardness of coal and rock is put forward. The stress of roller cutter pick, via the rocker retarding mechanism, is delivered to drag torque of cutting motor output shaft. Mathematical model between the shearer motor working parameters and the hardness of coal and rock is established, by analysis of the stress combined with the mechanical model of the pick cutting coal and rock. It provides mathematical basis about the test data to verify the result of the experiment.
\end{abstract}

\section{Introduction}

In order to realize the technology of automatic height-adjustment of shearer drum in coal mine fully mechanized working face, a lot of research about automatic identification method of coal rock interface has carried out both at home and abroad. in which the method and technology of monitoring coal and rock interface based on the change of cutting motor current is presented[1 4]. Also the method of monitoring shearer cutting motor and traction motor under the condition of laboratory simulation to identify coal and rock interface is put forward.

At present, there is a certain research foundation about the method of monitoring motor working parameters is used in the study of coal and rock character recognition. Usually coal and rock are different in shape, physical and mechanical properties. And as there is stratification (layered surface formed naturally in sedimentary minerals) and jointing (shear fracture surface formed by the function of various geological forces) in coal seam structure, so the nature is different in all parts of the coal seam. These natural factors make shearer cutting device bear dynamic load and suffer the influence of load randomness in the process of breaking coal.

Study on the mathematical relationship between shearer cutting motor parameters and coal and rock hardness. Mathematical relationship formula of them is established by analysis of coal and rock resistance, transmission efficiency of rocker retarding mechanism and output torque of cutting motor.

\section{Coal and Rock Breaking Mechanism}

Cutting Properties of Coal and Rock. Coal seam contains different ingredients of rocks and hard inclusion, which have great different in physical characteristics. Especially reflected in hardness or soundness. As this feature is a kind of aggregative indicator to show the complexity of coal and rock crushing, so it is performance putting effect of tension, shear, bending and thermal force that resistance by coal and rock all together.

The firmness coefficient of coal and rock f, also known as Protodyakonov coefficient, was put forward by Protodyakonov a Soviet union scholars in 1926. It can be used to measure the firmness coefficient by using the stamp method and to calculate Protodyakonov coefficient. The ratio of the uniaxial compressive strength and compressive strength of dense clay (10MPa) expresses the Protodyakonov coefficient of rock. That is: 


$$
f=\frac{\delta_{c}}{10} K
$$

where $\mathrm{K}$ is conversion coefficient; $\delta_{c}$ is the uniaxial compressive strength of rock.

The firmness coefficient is used to classify the rock and the coal seam. $f \leq 4$ is coal and soft rock, $f=4 \sim 8$ is medium hard rock, $f \geq 8$ is hard rock, and the hardest rock $f$ reach 20 . At the same time, $f \leq 1.5$ is soft coal, $f=1.5 \sim 3.0$ is medium hard coal and $f \geq 3$ is hard coal.

Breaking Mechanism of Shearer. When shearer cutting, the load on cutting picks is uneven, different hardness of coal and rock caused different cutting resistance. The load of roller and cutting and dragging motor is transmitted by the retarding mechanism and traction drive mechanism of the rocker. Some of the parameters will change when shearer cutting from idling to coal seam and to rock, for instance, electric current and velocity of cutting motor.

So the distinguish of coal and rock interface can be found by working parameters of cutting motor, referring to the characteristic values of coal and rock under different conditions in the same fully mechanized face.

\section{Theoretical Analysis between Working Parameter and Hardness of Coal and Rock}

The three-phase squirrel cage induction explosion-proof motor YBCS4-400, whose power is $400 \mathrm{KW}$, is used in cutting department. According to the condition of specific working environment of motor in mine, the motor must be guaranteed to be anti-explosion machine and electric spark-proof, to ensure absolutely security. And reliable work, big starting torque, strong overload capacity and high efficiency are also needed by motor.

In general shearer cutting motor is three-phase asynchronous motor, and its input power calculation method as follows:

$$
P=\sqrt{3} U_{L} I_{L} \cos \varphi
$$

where $U_{L}$ is line voltage of cutting motor; $I_{L}$ is line current of cutting motor; $\cos \varphi$ is power factor of cutting motor.

Ignore the stator copper loss, the cutting motor electromagnetic torque approximate is the output torque:

$$
T_{e}=\frac{P}{\omega}
$$

where $\omega$ is cutting motor speed ( $\omega=2 \pi f / p_{n}, f$ is frequency range, $p_{n}$ is number of pole-pairs).

In general cutting state, the relationship between electromagnetic torque and the resistance torque of the cutting motor is

$$
\eta K_{p} T_{e}=T_{L}
$$

where $T_{L}$ is the resistance torque; $\eta$ is mechanical transmission efficiency, which is constant (as shown in figure 1 ); $K_{p}$ is the motor load coefficient, which is constant (the ratio of real power and rated power).

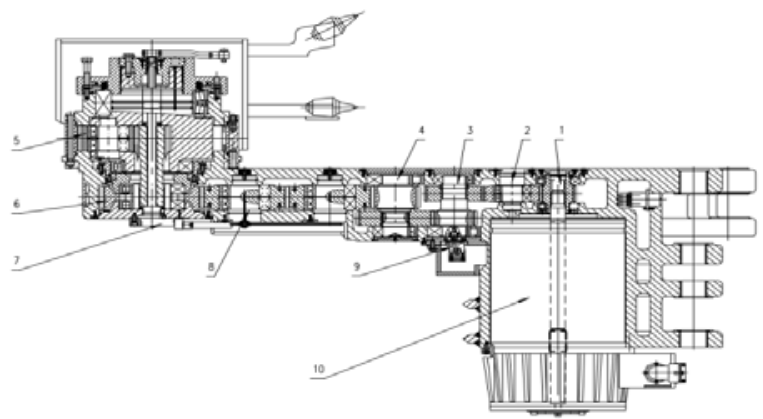

1--the first axis; 2--the second axis; 3--the third axis; 4--the fourth axis; 5--planetary mechanism; 6--big gear wheel; 7-- the spray water supply device(left); 8--the fifth axis; 9--STAUFF gear pump; 10--YBCS4-400/3.3KV motor

Fig1 The shearer drum rocker transmission organization charts 
Mechanical transmission efficiency $\eta$ depends on the transmission way and characteristics of cutting department. In this part, multistage gear transmission efficiency is a major consideration. The shearer MG400/940-WD is studied in the paper, and the structure is motor-- rocker-- planet gear-- roller. This transmission way adopts three-phase asynchronous motor with the longitudinal output shaft, and its efficiency of experience value is $91.5 \%$. The gear transmission efficiency is related to gear type, working accuracy and lubrication situation, so it can be got in list according to working accuracy and lubrication situation. The transmission efficiency is 0.97 for a general seven cylindrical gear. Normally the transmission efficiency is 0.9-0.99 for a cylindrical spur gear, 0.97 for a general eight cylindrical gear, and 0.98-0.99 for rolling bearing.

The tangential force of cutting motor at roller is:

$$
F=\frac{T_{L}}{R_{\text {head }}}=\frac{\eta K_{p} T_{e}}{R_{\text {head }}}
$$

where $F$ is tangential force of roller (cutting action of roller is shown in figure 2 ); $R_{\text {head }}$ is mean radius of roller (as shown in figure 2).
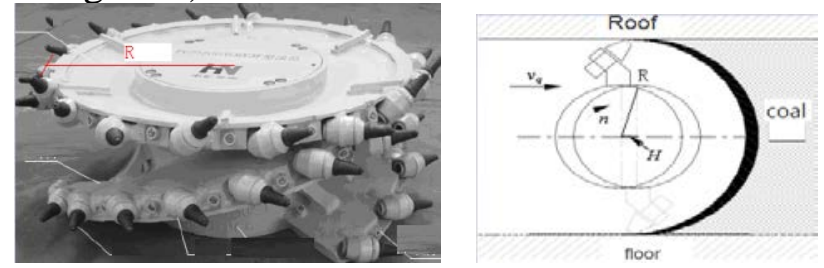

Fig2 Shearer drums radius and cutting action diagram

When the roller cutting coal and rock, the pressure intensity of tangential force to coal and rock at rotation direction is:

$$
P_{a}=\frac{F}{A}
$$

where $P_{a}$ is pressure intensity suffered by coal and rock; $A$ is the sum of the projection area of picks that participate in cutting (as shown in fig3).
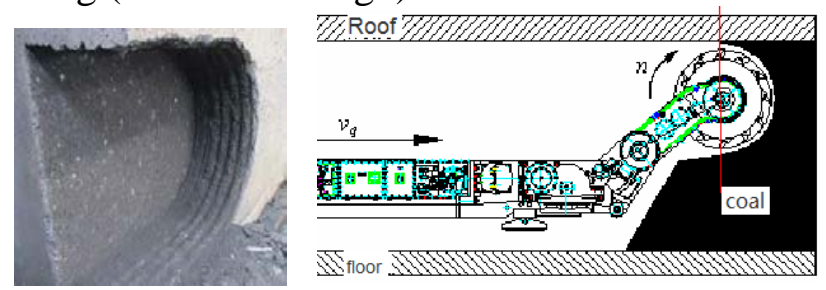

Fig3 Shearer involved in cutting cutter diagram

When roller cutting along direction of rotation, the relationship between uniaxial compressive strength of rock and the pressure intensity is:

$$
\delta_{c} \approx P_{a}
$$

From formula 1.1 to 2.6, the following relationships can be inferred:

$$
\begin{aligned}
& f=\frac{K}{10} \delta_{c}=\frac{K}{10} \times \frac{\sqrt{3} U_{L} I_{L} \eta K_{p} \cos \varphi}{\omega R_{\text {head }} A} \\
& C_{\text {cuttingmotor }}=\frac{\eta \cos \varphi}{\omega R_{\text {head }} A}, f=\frac{K}{10} \times C_{\text {cuttingmotor }} K_{\mathrm{p}} \sqrt{3} U_{L} I_{L} .
\end{aligned}
$$

From formula 2.7, line voltage $U_{L}$ and line current $I_{L}$ of cutting motor are collected and recorded by shearer black box, power factor $\cos \varphi$ is determined by the factory parameter and the load coefficient $K_{P}$ is constant (the ratio of real power and rated power). Mechanical transmission efficiency $\eta$, cutting motor speed $\omega$ ( $\mathrm{rad} / \mathrm{s})$, mean radius of roller $R_{\text {head }}(\mathrm{m})$ and the sum of the projection area of picks $A$ that participate in cutting can got in instruction book.

\section{Data Analysis}

(1) Cutting motor parameters

Take shearer MG400/940-WD as an example, power factor $\cos \varphi$ is determined by the factory 
parameter and the load coefficient $K_{P}$ is constant (the ratio of real power and rated power). Mechanical transmission efficiency $\eta$, cutting motor speed $\omega$ (rad/s), mean radius of roller $R_{\text {head }}$ (m) and the sum of the projection area of picks $A$ that participate in cutting can got in instruction book. The three-phase squirrel cage induction explosion-proof motor YBCS4-400 is used in cutting department. There is involute spline in the output shaft of the motor, through which transmit output power to gear deceleration mechanism of rocker.

(2) Determine the revolving speed $n$, power $P$ and torque $T$ of each shaft

1) Determine the revolving speed $n$ of each shaft

$$
\begin{aligned}
& n_{1}=n_{\text {motor }}=1470 \mathrm{r} / \mathrm{min} \\
& n_{2}=n_{1}=1470 \mathrm{r} / \mathrm{min} \\
& n_{3}=n_{2} / i_{1}=1470 / 1.77=830 \mathrm{r} / \mathrm{min} \\
& n_{4}=n_{3} / i_{2}=830 / 1.76=472 \mathrm{r} / \mathrm{min} \\
& n_{5}=n_{4}=472 \mathrm{r} / \mathrm{min} \\
& n_{6}=n_{5} / i_{3}=472 / 2.238=211 \mathrm{r} / \mathrm{min} \\
& n_{7}=n_{6} / i_{\text {sphere }}=211 / 6=35 \mathrm{r} / \mathrm{min}
\end{aligned}
$$

2) Determine the import rated power $P$ of each shaft

$$
\begin{aligned}
& P_{1}=400 \mathrm{~kW} \\
& P_{2}=P_{1}=400 \mathrm{~kW} \\
& P_{3}=P_{2} \times \eta_{1} \times \eta_{2}=400 \times 0.97 \times 0.99=384 \mathrm{~kW} \\
& P_{4}=P_{3} \times \eta_{1} \times \eta_{2}=384 \times 0.97 \times 0.99=369 \mathrm{~kW} \\
& P_{5}=P_{4}=369 \mathrm{~kW} \\
& P_{6}=P_{5} \times \eta_{1} \times \eta_{2}=369 \times 0.97 \times 0.99=354 \mathrm{~kW} \\
& P_{7}=P_{6}=354 \mathrm{~kW}
\end{aligned}
$$

where $\eta_{1}$ is gear mesh efficiency, $\eta_{1}=0.97 ; \eta_{2}$ is the efficiency of bearing, $\eta_{2}=0.99$.

The specific parameters of empirical formula 2.7 is got after the above calculation, as shown in tab. 1 and formula 3.1.

Tab.1 The parameters specific values of the empirical formula

\begin{tabular}{|r|c|c|c|c|}
\hline$\eta$ & $\cos \varphi$ & $\omega_{\mathrm{rad} / \mathrm{s}}$ & $R_{\text {Read }}(\mathrm{m})$ & $A\left(\mathrm{~m}^{2}\right)$ \\
\hline 0.885 & 0.85 & $49 \pi$ & 0.9 & $0.021 \pi$ \\
\hline \multicolumn{5}{c}{$C_{\text {cutting motor }}=\frac{\eta \cos \varphi}{\omega R_{\text {head }} A}=0.08197$}
\end{tabular}

The power factor $\cos \varphi$ is changed by load change, the standard value is always power factor under full load. In general, power factor will be more smaller as the load is more lower. The change of the cutting motor working parameters under high load, when roller cutting the roof and floor rock stratum, is detailed studied in this paper.

According to actual work experience, overload condition of cutting motor is infrequent. So power factor can take the standard value for simplified calculation, under the condition without affecting the purpose of research and combine with actual situation.

From formula $f=\frac{K}{10} \times C_{\text {cutting motor }} K_{p} \sqrt{3} U_{L} I_{L}$, the relationship between motor power of cutting motor and Protodyakonov coefficient is got. Substitute it into tab. 1, then get the formula 3.2:

$$
f=\frac{K}{10} \times 0.075 \times \sqrt{3} U_{L} I_{L}
$$

where $K_{p}$ approximate is the motor YBCS-400 rated efficiency 0.915.

\section{Conclusions}

By monitoring the cutting motor voltage and current, the output power and output torque of motor are obtained. The mathematical model of voltage and current of the motor is finally calculate, 
based on transfer efficiency of multilevel retarding mechanism of the rocker.

There is strong practical significance to identify the hardness of coal and rock automatically, and the foundation is accumulated in this paper.

\section{Acknowledgement}

In this paper, the research was sponsored by the The National Basic Research Program of China 973 Program (Project No. 2014CB046306) and Youth Fund Project of CUMTB (Project No. 800015FC).

\section{References}

[1] Sun Jiping, SHE Jie. Coal- rock imagefeature extraction and recognition based on support vector machine [J]. Journal of China Coal Society, 2013, 38(S2)

[2] WANG Yu- feng, XIA Yuan-tao, WANG Xiao-chen. Application on overcomplete ICA with noise in coal and rock identification of fully mechanized mining [J]. Journal of China Coal Society, 2011.05.36 (S1)

[3] Liu Chun-sheng, Yang Qiu, Li Chun-hua. Simulation of shearer drum cutting with memory program controlling by fuzzy control[J]. Journal of China Coal Society , 2008(07).33(07):822-825.

[4] Liu Chun sheng, Jing Kai, Wan Feng. Simulation of memory programmed hydraulic control system for height adjustment of shearer's drum [J]. Chinese Journal of Construction Machinery. 2007.04.5 (02): :142-146.

[5] Wang Zengcai, Fu Qiang. Attenuation of natural $\Gamma$ ray passing throughcoal seam and hydraulic support [J]. Journal of Liaoning Technical University. 2006.06, 25(06):804-807.

[6] Wang Zengcai, Meng Huirong. Influence of Canopy of Hydraulic Support upon Measurting Thickness of Coal Seam by Natural Gamma Ray[J]. Journal of China University of Mining \& Technology. 2002.05.31(3):323-326.

[7] Liang Yiwei, Xiong Shibo. Forecast of coal-rock interface based on neural network and dempster-shafter theory[J]. Journal of China Coal Society, 2003,28(01):86-90.

[8] Liu Junli, Zhao Haojie, Li Changyou. Coal-Rock Recognition Method Based on Cutting Vibration Features of Coal Shearer Drums[J]. Coal Science and Technology. 2013,10.41(10):93-95.

[9] Reng Fang. Study on the theory and method of coal-rock interface recognition based on multi-sensor Data Fusion technique[D]. Taiyuan University of Technology.2003.

[10] Yang Jianjian, Xue Guanghui, Wu Miao. Research and Development on Mine Intrinsic Safe Mode Vibration Sensor[J]. Coal Science and Technology. 2013,41(02): 71-74 